\title{
Satisfacción de la recuperación anestésica postoperatoria por la escala de lowa
}

\author{
Idoris Cordero-Escobar ${ }^{1 *}$, Aymé González-Torrero ${ }^{1}$ y Gisela Pérez-Martínez ${ }^{2}$ \\ ${ }^{1}$ Servicio de Anestesiología y Reanimación; ²Departamento de Enseñanza. Hospital Hermanos Ameijeiras, La Habana, Cuba
}

\begin{abstract}
Resumen
Introducción: La calidad de la asistencia generalmente se evalúa en función de los resultados y cuenta con estándares bien definidos. Objetivos: Determinar el grado de satisfacción de la recuperación anestésica según la escala de lowa. Métodos: Se realizó un estudio descriptivo, de corte transversal, para evaluar la satisfacción de los pacientes en el postoperatorio después de un procedimiento anestésico electivo. Se encuestaron con la Escala de lowa, 710 pacientes. Resultados: Predominó el grupo etario entre 29 y 39 años (38\%), el sexo masculino (63\%) y el estado físico ASA II (63\%). Los procedimientos quirúrgicos más frecuentes fueron los de cirugía general (35\%); el tiempo quirúrgico, entre 60-120 minutos (48\%), y la técnica anestésica, la anestesia general (56\%). No se presentaron complicaciones intraoperatorias en el $85 \%$ de los casos. Entre las complicaciones postanestésicas más frecuentes primaron los temblores (27\%). La puntuación media en la escala de lowa según satisfacción del paciente fue $4.05 \pm 0.75,5.75 \pm 1.25$ y 7.15 \pm 1.70 , y su correlación cuando la satisfacción fue baja, moderada y alta, respectivamente. Conclusiones: Se constató un alto grado de satisfacción de la recuperación anestésica en intervenciones quirúrgicas electivas. El valor de la puntuación de la escala de lowa midió de forma concreta la correlación entre el valor numérico y el alto grado de satisfacción de la recuperación anestésica.
\end{abstract}

Palabras clave: Anestesia. Satisfacción del paciente. Complicaciones.

\section{Satisfaction of postoperative anesthetic recovery by the lowa test}

\section{Abstract}

Introduction: The quality of assistance is generally evaluated based on the results and has well-defined standards. Objective: To determine the degree of satisfaction recovery anesthetic, according to the lowa Scale. Methods: A descriptive, cross-sectional study was conducted to assess the satisfaction of patients in the postoperative period after receiving an anesthetic procedure, electively. 791 patients were surveyed with the lowa Scale. Results: The age group prevailed between 29 and 39 years (38\%), the male sex (63\%) and the physical state ASA II (63\%). The most frequent surgical procedures were those of General Surgery (35\%), the surgical time between 60-120 minutes in 48\%. The most common anesthetic technique was general anesthesia 56\%. There were no intraoperative complications in $85 \%$ and post-anesthesia were tremors in $27 \%$ of the total. The average score on the lowa Scale according to patient satisfaction was $4.05 \pm$ $0.75,5.75 \pm 1.25$ and $7.15 \pm 1.70$ and its correlation with satisfaction was low, moderate and high respectively. Conclusions:

Correspondencia:

*Idoris Cordero-Escobar E-mail: ice@infomed.sld.cu
Disponible en internet: 17-09-2020 Rev Argent Anestesiol. 2019;77(2):44-50 www.revistaargentinaanestesiologia.com 0370-7792 @ 2020 Federación Argentina de Asociaciones, Anestesia, Analgesia y Reanimación. Publicado por Permanyer México SA de CV. Este es un artículo open access bajo la licencia CC BY-NC-ND (http://creativecommons.org/licenses/by-nc-nd/4.0/). 
A high degree of satisfaction with the anesthetic recovery was observed, which was verified electively. The value of the lowa Scale score, specifically measured the correlation between numerical value and the high degree of satisfaction of anesthetic recovery.

Key words: Anesthetic. Patient satisfaction. Complication.

\section{Introducción}

La calidad de la asistencia generalmente se evalúa en función de los resultados y cuenta en la actualidad con estándares bien definidos de morbilidad y mortalidad ${ }^{1-3}$.

La necesidad de documentar y evaluar la condición física de los pacientes a su llegada a la sala de recuperación postanestésica resulta del patrón desordenado de índices que incluyen los signos vitales, las complicaciones y si se utilizó o no una vía respiratoria artificial. La falta de unidad de criterios hizo aparente el requerimiento de establecer un sistema puntual y reconocido que indique la condición de llegada del paciente, su progreso hacia la recuperación de reflejos y consciencia y sobre todo si los pacientes pueden ser trasladados a la zona de hospitalización, donde el cuidado de enfermería es menos intenso y donde la valoración es menos frecuente ${ }^{3}$.

La evaluación de la satisfacción de los pacientes, luego de cualquier procedimiento anestésico, es un importante parámetro para el control de la calidad y mejoramiento continuo de la atención hospitalaria. La finalidad principal del cuidado anestésico es proporcionar a los pacientes seguridad y satisfacción durante los diferentes procedimientos ${ }^{2-4}$.

Sin embargo, para Rando, et al. ${ }^{1}$, la valoración de acuerdo a los resultados deja de lado la experiencia subjetiva del paciente. En el área de recuperación anestésica diversos estudios han resaltado su importancia, toda vez que constituye un reflejo de la calidad de asistencia anestesiológica.

Las encuestas de calidad y de satisfacción muestran el proceso asistencial desde la perspectiva del propio paciente. Este enfoque no sustituye la guía clásica con base en los resultados, sino que es complementario, pues apunta a aspectos diferentes, más subjetivos y menos técnicos ${ }^{2}$.

Dentro de las escalas evaluativas, se han desarrollado diferentes medidas con el fin de evaluar la calidad de la visita preoperatoria del anestesiólogo. Esta herramienta evaluó la satisfacción con la visita preoperatoria y la información sobre el paciente obtenida como resultado de la misma. Este estudio se realizó en Alemania y su validez e idoneidad cuando se tradujo a otros idiomas aún no se ha implantado ${ }^{5-9}$.
Chanthong, et al..$^{10}$ evaluaron la consulta preoperatoria y su relación con la empatía. Para ello aplicaron una herramienta que se había desarrollado y validado previamente para evaluar la satisfacción del paciente en la consulta en atención primaria.

Neuman ${ }^{11}$ señaló que el Grupo de Enlace del Paciente del Real Colegio de Anestesiólogos del Reino Unido propuso un instrumento para determinar la validez donde se calculó la fiabilidad generalizada y la consistencia interna. Refirió que Auquier construyó una herramienta que consistió en un cuestionario para evaluar la satisfacción del paciente durante la atención perioperatoria y concluyó que el cuestionario fue una valiosa ayuda para evaluar las opiniones de los pacientes en el periodo perioperatorio.

Caljouw, et al. ${ }^{7}$ realizaron un protocolo riguroso en el que se incluyó un cuestionario psicométrico para determinar la satisfacción de la atención durante la anestesia y concluyeron que debe incluir áreas relacionadas con la información, con la participación en la toma de decisiones y con el anestesiólogo. Describieron, además, que, durante un periodo de 5 años, Hüppe realizó tres estudios en los que evaluó un nuevo cuestionario perioperatorio conocido como Cuestionario anestesiológico y lo adaptó para su uso en anestesia cardíaca, con mejor evaluación psicométrica en esta cohorte de pacientes.

Se han realizado diferentes escalas evaluativas de la calidad y la satisfacción de la recuperación postanestésica ${ }^{1}$, la satisfacción y el tiempo de recuperación con diferentes técnicas anestésicas ${ }^{2}$, la satisfacción según la escala de lowa ${ }^{3,4}$, la escala de calidad de la recuperación postoperatoria ${ }^{5-7}$, así como revisiones sistemáticas ${ }^{5-9}$, que identificaron un gran número de cuestionarios psicométricos que se han desarrollado para medir la satisfacción del paciente con anestesia en una variedad de especialidades clínicas y contextos.

Sin embargo, de más de 3,000 artículos que utilizaron la satisfacción del paciente en el periodo perioperatorio como medida de resultados, solo 71 utilizaron medidas de satisfacción. Los principales señalamientos fueron que no incluían aspectos multidimensionales, ni incluían ningún tipo de proceso de exploración psicométrica y que la gran mayoría de los estudios relacionados con la anestesia no utiliza herramientas validadas 
para medir la satisfacción, y se cree que este resultado sería de gran importancia ${ }^{10-15}$.

Giménez García, et al. ${ }^{5}$ validaron en oftalmología la versión al español de la escala de lowa. La omisión alguna respuesta del cuestionario (Tabla 1) puede dar lugar a sesgos, por lo que se debe alentar a los médicos e investigadores a la incorporación de escalas validadas en la práctica diaria y en estudios clínicos ${ }^{16-20}$.

Esta valoración cualitativa debe proporcionar una guía para los anestesiólogos, revisores y editores sobre las medidas que están disponibles y que son válidas y, por tanto, aumentar los estándares de informes de resultado en los estudios académicos y mejorar la calidad en la práctica clínica anestesiológica ${ }^{13-15}$.

Constituye el objetivo de esta investigación determinar el grado de satisfacción, según la escala de lowa, de la recuperación anestésica postoperatoria en pacientes intervenidos quirúrgicamente con algún tipo de anestesia.

\section{Diseño metodológico}

Se realizó un estudio descriptivo, de corte transversal, con el fin de evaluar la satisfacción de los pacientes en el postoperatorio inmediato después de un procedimiento anestésico de forma electiva en el Hospital Clínico Quirúrgico Hermanos Ameijeiras entre mayo del 2016 y abril del 2019.

\section{Universo de estudio}

A los pacientes programados para cualquier procedimiento quirúrgico electivo con anestesia y que una vez finalizado el proceder quirúrgico se trasladaron a la unidad de cuidados postanestésicos (UCPA), se les aplicó la escala de lowa y cumplieron los criterios de selección.

\section{Criterios de inclusión}

Pacientes entre 18 y 60 años, de ambos sexos y cualquier color de piel, con estado físico según la Sociedad Americana de Anestesiología (ASA) I a III, que requirieron anestesia general o regional para cualquier procedimiento quirúrgico electivo y que firmaron el modelo de consentimiento informado con el que aceptaron formar parte del estudio.

\section{Criterios de exclusión}

Pacientes que fueron trasladados a la unidad de cuidados intensivos, cuidados intensivos coronarios 0 tuvieran un desenlace fatal.
Tabla 1. Escala de lowa

\begin{tabular}{|l|l|}
\hline 1 & Vomité o tuve ganas de vomitar \\
\hline 2 & Me gustaría recibir la misma anestesia otra vez \\
\hline 3 & Sentí picazón \\
\hline 4 & Me sentí relajado \\
\hline 5 & Sentí dolor \\
\hline 6 & Me sentí seguro \\
\hline 7 & Tuve mucho frío o calor \\
\hline 8 & Quedé satisfecho con mi cuidado anestésico \\
\hline 9 & Sentí dolor durante la cirugía \\
\hline 10 & Me sentí bien \\
\hline 11 & Me sentí lastimado \\
\hline
\end{tabular}

Tabla 2. Posibilidades de respuesta para la escala de lowa como se utiliza en los estudios en el idioma inglés y su respectivo puntaje

\begin{tabular}{|l|c|}
\hline Totalmente en desacuerdo & 3 \\
\hline Moderadamente en desacuerdo & 2 \\
\hline Levemente en desacuerdo & 1 \\
\hline Levemente de acuerdo & 1 \\
\hline Moderadamente de acuerdo & 2 \\
\hline Totalmente de acuerdo & 3 \\
\hline
\end{tabular}

\section{Muestra}

Quedó conformada por 710 pacientes programados para cualquier procedimiento quirúrgico electivo que requirieron anestesia y que una vez finalizado el proceder quirúrgico se trasladaron a la UCPA para evaluar la satisfacción de los pacientes en el postoperatorio entre mayo del 2016 y abril del 2019. Se encuestaron con la Escala de lowa 710. A todos los pacientes se les realizó la encuesta que evalua 11 items y las posibles respuestas.

\section{Variables}

La variable principal fue la satisfacción del paciente con el tratamiento anestésico. Las variables secundarias intraoperatorias fueron el procedimiento quirúrgico, la técnica anestésica y el tiempo quirúrgico, y las variables postoperatorias, la analgesia de rescate y las complicaciones del procedimiento quirúrgico y anestésico. Las variables generales fueron la edad, el sexo y la clasificación de ASA. 
A todos los pacientes se les llenó el siguiente formulario (Tabla 1) y sus respuestas (Tabla 2).

Para cada uno de los acápites existe un patrón de respuesta politómica de 6 posibilidades de tipo numérico con un puntaje de $-3 a+3$ que permite el análisis cuantitativo. Todas las afirmaciones están escritas con sentido positivo, como por ejemplo, «Yo me siento bien». Si se responde con una de las opciones «De acuerdo", implicaría satisfacción con la anestesia. Para las afirmaciones escritas con sentido negativo, si se responde con una opción «De acuerdo", se estaría expresando insatisfacción con la anestesia. Un paciente totalmente satisfecho tendrá un puntaje de 3 , que es el máximo posible. La satisfacción se clasificó como de bajo grado si el valor medio de la escala de lowa era $<5$; como moderada si era entre 5.5 y 6.0 , y de alto grado si era $\geq 7.0$.

\section{Procesamiento y análisis estadístico de los datos}

Para el análisis estadístico se utilizaron medidas de resumen para variables cualitativas (porcentajes) y cuantitativas (media y desviación estándar). Para la información obtenida se utilizó una base de datos en Excel y se procesó con el programa estadístico SPSS versión 20.

\section{Ética y seguridad}

A todos los pacientes se les explicó en qué consistía la investigación y qué proceder anestésico se emplearía durante la operación, así como la privacidad que se mantendría con todos los datos empleados. Se le explicó, además, que si no querían participar en la investigación, ello no influiría ni en su tratamiento ni en la relación médico-paciente. Se les comunicó su derecho a abandonar la investigación cuando lo estimaran pertinente. Cada paciente disfrutó de una vigilancia estrecha con todo el sistema de monitorización disponible en el quirófano, participara o no en la investigación. Los pacientes que aceptaron participar en el estudio dieron su consentimiento firmando el modelo de consentimiento del paciente por escrito y se aprobó por el Comité de Ética del Hospital.

\section{Resultados}

Se estudiaron 710 pacientes. Con relación a las variables generales, predominó el grupo etario entre 29
Tabla 3. Distribución de pacientes según las complicaciones postanestésicas inmediatas

\begin{tabular}{|l|c|c|}
\hline Proceder & Número & $\%$ \\
\hline Bloqueo residual & 5 & 0.7 \\
\hline Disrritmias & 7 & 1 \\
\hline Hipoxemia & 26 & 4 \\
\hline Somnolencia & 55 & 8 \\
\hline Hipertensión & 70 & 9 \\
\hline Dolor & 85 & 12 \\
\hline Medio interno & 115 & 16 \\
\hline Náuseas y vómitos & 130 & 18 \\
\hline Temblores & 190 & 27 \\
\hline
\end{tabular}

Tabla 4. Media y desviación estándar de la puntuación según el grado de satisfacción de los pacientes en la recuperación anestésica

\begin{tabular}{|l|c|c|c|}
\hline Satisfacción & NNúmero & $\%$ & Media (DE) \\
\hline Baja & 40 & 6 & $4.05(0.75)$ \\
\hline Moderada & 110 & 15 & $5.75(1.25)$ \\
\hline Alta & 560 & 79 & $7.15(1.70)$ \\
\hline Total & 710 & 100 & $8.95(2.05)$ \\
\hline
\end{tabular}

y 39 años $(n=270$; $38 \%)$, el sexo masculino $(n=450$; $63 \%$ ) y el estado físico ASA II ( $n=450 ; 63 \%)$.

El procedimientos quirúrgicos más frecuente fueron las intervenciones de cirugía general ( $n=250 ; 35 \%)$, seguidos por los de cirugía estética $(n=162 ; 23 \%)$ y por procedimientos ortopédicos $(n=100 ; 14 \%)$. La media del tiempo quirúrgico en la mayoría de los pacientes osciló entre 60-120 minutos $(n=340 ; 48 \%)$. La técnica anestésica que predominó fue la anestesia general $(n=400 ; 56 \%)$ versus regional $(n=310 ; 44 \%)$.

No se presentaron complicaciones intraoperatorias en la mayoría de los pacientes $(n=600 ; 85 \%)$. Las complicaciones postanestésicas inmediatas se muestran en la tabla 3. Predominaron los temblores $(27 \%$ del total).

En la tabla 4 se observa la media y la desviación estándar $(X \pm D S)$ de la puntuación en la escala de lowa del total de pacientes según satisfacción del paciente en la recuperación anestésica. Las medias de los valores obtenidos al aplicar la escala de lowa fueron $4.05 \pm 0.75,5.75 \pm 1.25$ y $7.15 \pm 1.70$, y su 
correlación con la satisfacción fue baja, moderada y alta, respectivamente.

\section{Discusión}

Las guías prácticas son recomendaciones desarrolladas de forma sistemática para la toma de decisiones sobre los cuidados de salud. Estas recomendaciones pueden ser adoptas, modificadas o rechazadas de acuerdo a las necesidades clínicas del paciente y de las políticas institucionales. La ASA dejó bien definido que el desarrollo de guías de prácticas clínicas no son requerimientos absolutos, y su aplicación no constituye garantía de resultados especificos ${ }^{16-18}$.

Diversos artículos ${ }^{1-9,11,13,18-23}$ evaluaron la satisfacción en pacientes con diversos procedimientos quirúrgicos (oftalmológicos, estomatológicos, cirugía mayor ambulatoria, colonoscopias, por solo citar algunos). En esta investigación, la escala se aplicó en varias especialidades, pero la más frecuente fue en cirugía general (35\%), y la intervención quirúrgica más frecuente fue la colecistectomía laparoscópica (60\%). El tiempo quirúrgico influyó en la satisfacción, así como la técnica anestésica empleada. La media del tiempo quirúrgico osciló entre 120-180 minutos (48\%). La técnica anestésica más frecuente fue la anestesia general (56\%), lo que corrobora los resultados de otros autores ${ }^{24-30}$.

La evaluación de la calidad asistencial a través de resultados (morbilidad y mortalidad) deja de lado la experiencia subjetiva del paciente. En esta serie, la mayoría de los pacientes no tuvieron complicaciones (85\%). Las complicaciones postoperatorias más frecuentes fueron temblores (27\%), hipertensión arterial $(18 \%)$, dolor (12\%) y náuseas y vómitos $(9 \%)$. Resultados que concuerdan con otros autores ${ }^{31-34}$.

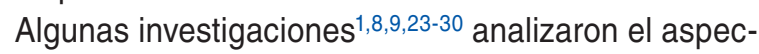
to subjetivo de la recuperación anestésica mediante una escala visual análoga de satisfacción (EVA) y un cuestionario dirigido a evaluar la calidad de la asistencia anestesiológica. Se entrevistaron a las 24 horas del alta de la UCPA 166 pacientes operados. A cada pregunta se le asignó un valor numérico y se construyó una escala global de calidad (QoR). Las preguntas se agruparon en función de cinco aspectos de la recuperación: satisfacción, emociones, independencia física, apoyo psicológico y dolor. El valor promedio de la QoR fue de $175.33 \pm 11.3$ (89.9\% del puntaje máximo). Los aspectos que mostraron valores más altos de puntuación fueron el apoyo psicológico y el dolor, y los más bajos la independencia física y la satisfacción. Las emociones obtuvieron un valor intermedio.
Existieron diferencias significativas entre los promedios de la QoR con relación al tipo de anestesia, complicaciones en el área de recuperación y estado físico (ASA III). El promedio del valor de la escala de satisfacción fue de $7.45 \pm 1.87$. Un $30 \%$ de la población señaló valores menores a 7 , un $38 \%$ marcó valores entre 7 y 8.5 y un $30 \%$ entre 9 y 10. La experiencia del paciente evaluada a través de la satisfacción global (EVA) y de la calidad de recuperación (QoR) constituye una medida cuantitativa de la calidad de recuperación postoperatoria y nos permite realizar un seguimiento del proceso de atención, identificando los aspectos que más se afectan por la cirugía y la anestesia en la población hospitalaria ${ }^{1,8,9,23}$.

La calidad de la asistencia generalmente se evaluó en función de los resultados. Se cuenta en la actualidad con estándares definidos de morbilidad y mortalidad 1 . Sin embargo, la valoración de acuerdo a los resultados deja de lado la experiencia subjetiva del paciente. En el área de recuperación anestésica diversos estudios han resaltado su importancia, pues constituye un reflejo de la calidad de asistencia anestesiológica ${ }^{2-5}$.

La satisfacción del paciente unida a marcadores objetivos de calidad se utiliza en la actualidad para valorar el proceso de atención $n^{6,7}$. La satisfacción se cuantifica mediante escalas numéricas o nominales que no permiten discernir cuáles son los aspectos positivos y negativos de la experiencia ${ }^{8-10}$. Los marcadores objetivos de calidad propuestos por Myles ${ }^{4,24,25}$ se elaboraron con base en cuestionarios sistemáticos que exploran en forma independiente los factores determinantes de la experiencia subjetiva.

Ferreira, et al. ${ }^{21}$ publicaron que son varios los factores que parecen influir en el grado de satisfacción de los pacientes, como la edad, el sexo, la ansiedad, la depresión, las náuseas y vómitos y el dolor postoperatorio. Ello concuerda con los hallazgos de esta investigación.

Tal como en estudios anteriores ${ }^{1,3,12}$, los pacientes presentaron grados elevados de satisfacción. La explicación se encuentra normalmente relacionada con las expectativas de los pacientes por el desconocimiento sobre anestesia. Se sabe que el $85 \%$ de los pacientes esperan una anestesia sin problemas ${ }^{3}$, que valoran la comunicación y la información y que sus principales preocupaciones son el no despertar de la anestesia, las náuseas y vómitos, el dolor postoperatorio, los recuerdos intraoperatorios dentro de las principales complicaciones ${ }^{7}$. Así, si no existe ninguna complicación perioperatoria, se espera que el paciente esté satisfecho con la anestesia. 
Una de las preocupaciones de los pacientes es la ansiedad previa al procedimiento quirúrgico. De modo que para disminuirla debe utilizarse siempre y cuando no esté prescrita la medicación ansiolítica. Según los resultados presentados en esta serie, dicha medicación fue eficaz en casi la totalidad de los pacientes; sin embargo, no se encontró ninguna relación entre el grado de satisfacción y el uso de medicación preanestésica. De este modo, y a pesar de ser una preocupación de los pacientes, no se puede afirmar que la ansiedad sea un factor que influya en el grado de satisfacción con relación al acto anestésico ${ }^{7}$. No obstante, en esta investigación se medicaron preoperatoriamente todos los pacientes con benzodiacepinas, antiinflamatorios no esteroideos (AINE) y profilaxis antiemética.

La escala de lowa fue desarrollada en la universidad del mismo nombre por Dexter, et al. en $1997^{3}$. Se describió que las ventajas de esta escala radican en la evaluación directa, que permite extraer datos cuantitativos, y en que es unidimensional, de tipo discriminativo compuesto por un instructivo corto. Tiene 11 acápites, todos con el mismo peso o importancia. Los acápites están escritos como afirmaciones, donde la primera expresa una sensación negativa, la segunda una sensación positiva y así sucesivamente intercala su sentido positivo o negativo hasta finalizar las afirmaciones. Este orden evita el sesgo de aquiescencia, definida como la tendencia de los sujetos a estar siempre de acuerdo con las preguntas o afirmaciones de la escala sin importar su contenido ${ }^{5}$.

A pesar de la subjetividad, la satisfacción de los pacientes se considera un buen indicador de la calidad de los cuidados anestésicos ${ }^{17-19,20,25,30}$.

La escala de lowa se empleó en esta serie en el $100 \%$ de los pacientes una vez que estuvieran recuperados. La media de los valores obtenidos al aplicarla fueron $4.05 \pm 0.75,5.75 \pm 1.25$ y $7.15 \pm 1.70$ y su correlación con la satisfacción fue baja, moderada y alta, respectivamente.

Del total de pacientes en esta serie, el $79 \%$ refirió un alto grado de satisfacción con la recuperación anestésica, lo que concuerda con los resultados de otras investigaciones ${ }^{23,31-36}$.

Se concluye que se constató un alto grado de satisfacción de la recuperación anestésica, constatado de forma electiva. El valor de la puntuación de la escala de lowa midió de forma concreta la correlación entre valor numérico y el alto grado de satisfacción de la recuperación anestésica.

\section{Conflicto de intereses}

Las autores declaran que no existen conflictos de intereses.

\section{Responsabilidades éticas}

Protección de personas y animales. Los autores declaran que para esta investigación no se han realizado experimentos en seres humanos ni en animales.

Confidencialidad de los datos. Los autores declaran que han seguido los protocolos de su centro de trabajo sobre la publicación de datos de pacientes.

Derecho a la privacidad y consentimiento informado. Los autores han obtenido el consentimiento informado de los pacientes y/o sujetos referidos en el artículo. Este documento obra en poder del autor de correspondencia.

\section{Bibliografía}

1. Rando K, Solla G, Sálice L, Lambrosquini N, Basigniani N, Lanzaro R, et al. Evaluación de la calidad y la satisfacción de la recuperación posanestésica de los pacientes operados en el Hospital de Clínicas. Anest Analg Reanim [Internet] 2003 (consultado: marzo de 2016);18(2). Disponible en: $12732003000200003 \&$ script=sci_arttext

2. Dexter F. lowa Satisfaction with Anesthesia Scale [Internet]. (Consultado: marzo de 2016. Disponible en: http://www.franklindexter.net/Contracts/ ContractHourly.docx.

3. Dexter F, Candiotti KA. Multicenter assessment of the lowa Satisfaction with Anesthesia Scale, an instrument that measures patient satisfaction with monitored anesthesia care. Anesth Analg. 2011;113(2):364-8.

4. Myles PS, Hunt OJ, Moloney JT. Postoperative 'minor' complications: Comparison between men and women. Anaesthesia. 1997;52:300-6.

5. Giménez García LF, Capera ADR. Validación al español de la escala "The lowa Satisfaction with Anesthesia Scale (ISAS)" para cuidado anestésico monitorizado en cirugía de oftalmología. Rev Colomb Anestesiol. 2014;42:272-80.

6. Barnett SF, Alagar RH, Grocott, B MPW, Savvas G, Dick JR, Moonesinghe SR. Patient-Satisfaction Measures in Anesthesia: Qualitative Systematic Review. Anesthesiology. 2013;119:452-78.

7. Caljouw MA, van Beuzekom M, Boer F. Patient's satisfaction with perioperative care: development, validation, and application of a questionnaire. Br J Anaesth. 2008;100:637-44

8. Royse CF, Chung F, Newman S, Stygall J, Wilkinson DJ. Predictors of patient satisfaction with anaesthesia and surgery care: a cohort study using the Postoperative Quality of Recovery Scale. Eur J Anaesth. 2013;30(3):106-10.

9. Royse CF, Newman S, Chung F. Development and feasibility of a scale to assess postoperative recovery: the Postoperative Quality Recovery Scale. Anesthesiology. 2010;113:892-905.

10. Chanthong P, Abrishami A, Wong J, Herrera F, Chun F. Systematic Review of Questionnaires Measuring Patient Satisfaction in Ambulatory Anesthesia. Anesthesiology. 2009;110:1061-7.

11. Neuman S. Patient satisfaction and value in anesthesia care. Anesthesiology. 2011;114:1019-20.

12. Mui WC, Chang CM, Cheng KF. Development and validation of the questionnaire of satisfaction with perioperative anesthetic care for general and regional anesthesia in Taiwanese patients. Anesthesiology. 2011;114:1064-75.

13. Aldrete JA, Wright W. Criterios para dar de alta. El puntaje de recuperación postanestésica. Rev Col Anest. 1996;24:305-12.

14. Aldrete JA. The postanesthesia recovery score revisited. J Clin Anesth. 1995;7:89-91.

15. Kennedy GD, Tevis CD, Kent KC. Is There a Relationship Between Patient Satisfaction and Favorable Outcomes? Ann Surg. 2014; 260(4):592-600.

16. Alshehri AA, Alomar YM, Mohammed GA, Al-Fozan MS, Al-Harbi MS Alrobai KA, et al. A survey on postanesthetic patient satisfaction in a university hospital. Saudi J Anaesth. 2015;9(3):303-5.

17. Escribano-Hernándezb A, García-Garraus JM, Hernández-García I. Eva- 
luation of satisfaction among relatives of mentally disabled patients who were users of a dental care protocol under general anaesthesia. Med Oral Patol Oral Cir Bucal. 2012;17(1):e83-8.

18. Apfelbaum JL, Silverstein JH, Chung FF, Connis RT, Fillmore RB, Hunt SE. Practice Guidelines for Postanesthetic Care: An Updated Report by the American Society of Anesthesiologists Task Force on Postanesthetic Care. Anesthesiology. 2013;118: 291-307.

19. Gebremedhn EG, Chekol WB, Amberbir WD, Flatie TD. Patient satisfaction with anaesthesia services and associated factors at the University of Gondar Hospital, 2013: a cross-sectional study. BMC Res Notes. 2015;8:377.

20. Watkins TJ, Bonds RL, Hodges K, Goettle BB, Dobson DAM, Maye JP. Evaluation of postprocedure cognitive function using three distinct stadard sedation regimens for endoscopic procedures. AANA J. 2014;82(2):133-9.

21. Ferreira T, Oliveira F, Arede MJ, VicoM. Evaluación de la satisfacción de los pacientes con la anestesia. Estudio observacional transversal. Actualidad Médica 2018. En línea. Consultado Julio 2018, URL https://www. actualidadmedica.es/archivo/2015/796/or05.html.

22. De los Ríos-Arellano JG, Cordero-Escobar I, Pérez-Martínez G, Mora-Díaz I. Satisfacción de la recuperación anestésica postoperatoria según escala en pacientes con anestesia general y neuroaxial. Rev Mexicana Anest. 2017;40(4): 264-72

23. Vargo J, Howard K, Petrillo J, Scott J, Revicki DA. Development and validation of the patient and clinician sedation satisfaction index for colonoscopy and upper endoscopy. Clin Gastroenterol Hepatol. 2009;7(2):156-62

24. Myles PS, Hunt JO, Nightingale CE, Fletcher H, Beh T, Tanil D, et al. Development and psychometric testing of a quality of recovery score after general anesthesia and surgery in adults. Anesth Analg. 1999;88:83-90.

25. Myles PS, Weitkamp B, Jones K, Melick J. Hensen S. Validity and reliability of a postoperative quality of recovery score: the QoR-40. Br J Anaesth. 2000;84(1):11-5.

26. Myles PS, Williams DL, Hendrata M. Patient satisfaction after anaesthesia and surgery: results of a prospective survey of 10811 patients. $\mathrm{Br}$ Anaesth. 2000;84:6-10.
27. Stark PA, Myles PS, Burke JA. Development and psychometric evaluation of a postoperative quality of recovery score: the QoR-15. Anesthesiology. 2013;118(6):1332-40.

28. Royse CF, Chung F, Newman S, Stygall J, Wilkinson DJ. Predictors of patient satisfaction with anesthesia and surgery care: a cohort study using the Postoperative Quality of Recovery Scale. Eur J Anaesth. 2013;30(3):106-10.

29. Chazapis M, Walker EM, Rooms MA, Kamming D, Moonesinghe SR Measuring quality of recovery-15 after day case surgery. $\mathrm{Br} \mathrm{J}$ Anaesth. 2016;116(2):241-8

30. Gornall BF, Myles PS, Smith CL, Burke JA, Leslie K, Pereira MJ, Bost JE, Kluivers KB, Nilsson UG, Tanaka Y, Forbes A. Measurement of quality of recovery using the QoR-40: a quantitative systematic review. $\mathrm{Br} J$ Anaesth. 2013;111(2):161-9.

31. Royse CF, Newman S, Chung F. Development and feasibility of a scale to assess postoperative recovery: the Postoperative Quality Recovery Scale. Anesthesiology. 2010;113:892-905.

32. Myles PS, Myles DB, Galagher W, MacDonald N, Dennis A. Minimal Clinically Important Difference for Three Quality of Recovery Scales. Aneshesiology. 2016;125:39-45.

33. Williams MR, McKeown A, Dexter F, Miner JR, Sessler DI, Vargo J, et al. Efficacy Outcome Measures for Procedural Sedation Clinical Trials in Adults: An Action. Systematic Review. Anesth Analg. 2016; 122(1):152-70.

34. Cascella M, Fusco R, Caliendo D, Granata G, Carbone D, Muzio MR, et al. Anesthetic dreaming, anesthesia awareness and patient satisfaction after deep sedation with propofol target controlled infusion: A prospective cohort study of patients undergoing day case breast surgery. Oncotarget. 2017;8(45):79248-56.

35. Cordero Escobar I, Soler Morejón C, Espinaco Valdés J, González Torrero AJ, Pérez Martínez G. Patient Satisfaction Assessment Scales in Postoperative Anesthetic Recovery. EC Anaesthesia. 2018; 4(2):51-6.

36. Rando K, Solla G, Sálice S, Lambrosquini D, Basigniani N, Lanzaro L. et al. Evaluación de la calidad y la satisfacción de la recuperación posanestésica de los pacientes operados en el Hospital de Clínicas. En línea. Consultado. Mayo 2018. URL: https://www.researchgate.net/publication/317448356. 